\title{
Pulsar Observations at the Ghana Radio Astronomy Observatory
}

\author{
T. W. Scragg ${ }^{1}$, B. W. Stappers ${ }^{1}$, R. P. Breton ${ }^{1}$, J. N. Smith ${ }^{2}$, \\ D. Adomako ${ }^{3}$, B. Duah Asabere ${ }^{3}$, J. O. Chibueze ${ }^{2}$ and K. Cloete ${ }^{2}$, \\ ${ }^{1}$ Jodrell Bank Centre for Astrophysics, University of Manchester, Manchester M13 9PL, UK. \\ ${ }^{2}$ SKA-SA, The Park, Park Road, Pinelands, Western Cape, SA. \\ ${ }^{3}$ Ghana Space Science and Technology Institute, P. O. Box LG80 Legon-Accra, Ghana. \\ email: thomas.scragg@postgrad.manchester.ac.uk
}

\begin{abstract}
In August 2017 a new radio telescope, the Ghana Radio Astronomy Observatory (GRAO), was officially inaugurated at Kuntunse, Ghana. The GRAO is a former satellite Earth station and now the first operational station in the African VLBI Network (AVN). The Jodrell Bank Centre for Astrophysics (JBCA), supported by the UK's STFC/Newton Fund, has developed a new pulsar timing system (Hebe) for the GRAO. We present some aspects of the design of Hebe and an outline of the first pulsar detection at GRAO.
\end{abstract}

Keywords. Pulsars, instrumentation: miscellaneous, telescopes (GRAO).

\section{GRAO and Pulsar Timing}

The GRAO is a former telecommunications satellite Earth station and it's conversion to a radio observatory was in part funded by South Africa and the African Square Kilometre Array partnership agreement. The GRAO has a fully steerable, 32 meter Cassegrain beam wave-guide design dish connected to a room temperature receiver and currently capable of observing at $5 \mathrm{GHz}$ and $6.7 \mathrm{GHz}$ with a bandwidth of up to $400 \mathrm{MHz}$. Once fully commissioned the GRAO will enable local users to undertake independent scientific studies and participate in international research programs.

A new pulsar data processing system, 'Hebe', will be installed at the GRAO in late 2017. Developed at JBCA, Hebe is a dual 8-core CPU enterprise workstation with two NVIDIA Maxwell class Titan X GPUs and 32 TB of disk space. The Hebe_Control program operates in automatic or manual mode and uses the PSRDADA $†$ tools for data acquisition and the DSPSR software suite for pulsar data analysis (van Straten \& Bailes (2011)). Hebe is designed to process in real-time the signal data from the current telescope back-end systems by using the massively parallel processing capabilities of GPUs.

In order to select a suitable GPU, over 32,000 processing trials were conducted using various combinations of bandwidth, frequency, output filterbank channels and dispersion measure. The testing process was based on the methodology described in van Straten \& Bailes (2011) and an extract of the key processing throughput results is shown in Figure. 1. Hebe is also designed to be capable of commensal observations, for example, real time FRB searching during pulsar observations.

A number of pulsar science projects have already been identified for the GRAO. For instance, systematic observations of known pulsars in the $5-7 \mathrm{GHz}$ band, for which data are sparsely available, in order to study the evolution of pulse profiles and fluxes. Other projects include studies of pulsar timing noise, glitches and breaking indices.

$\dagger$ http://psrdada.sourceforge.net/index.shtml 

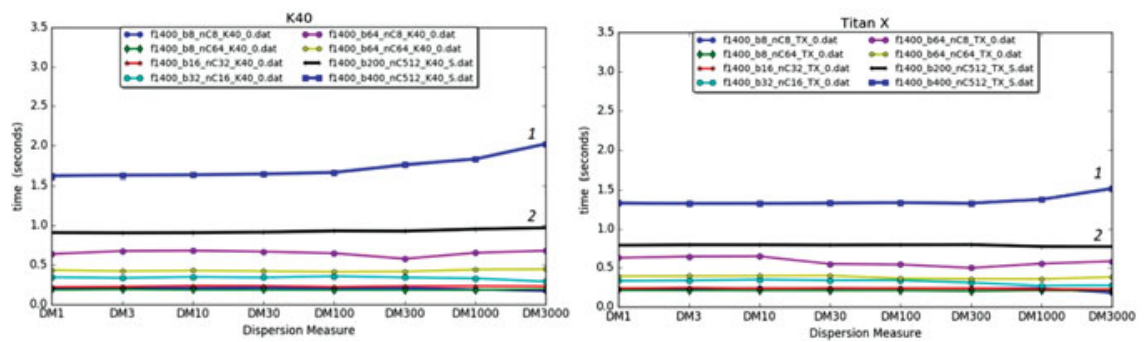

Figure 1. Processing time for $1 \mathrm{~s}$ of sample pulsar data with various input bandwidths and pulsar timing options. The left-hand plot is for a Tesla class K40 Scientific GPU and the right-hand plot is a Maxwell class Titan X consumer GPU. Line (1) on the plots shows that it takes greater than $1.5 \mathrm{~s}$ to process $400 \mathrm{MHz}$ bandwidth data and line (2) shows that $200 \mathrm{MHz}$ input data can be processed in under $1 \mathrm{~s}$. The vertical axis is the average time taken to process $1 \mathrm{~s}$ of data and the horizontal axis is dispersion measure.
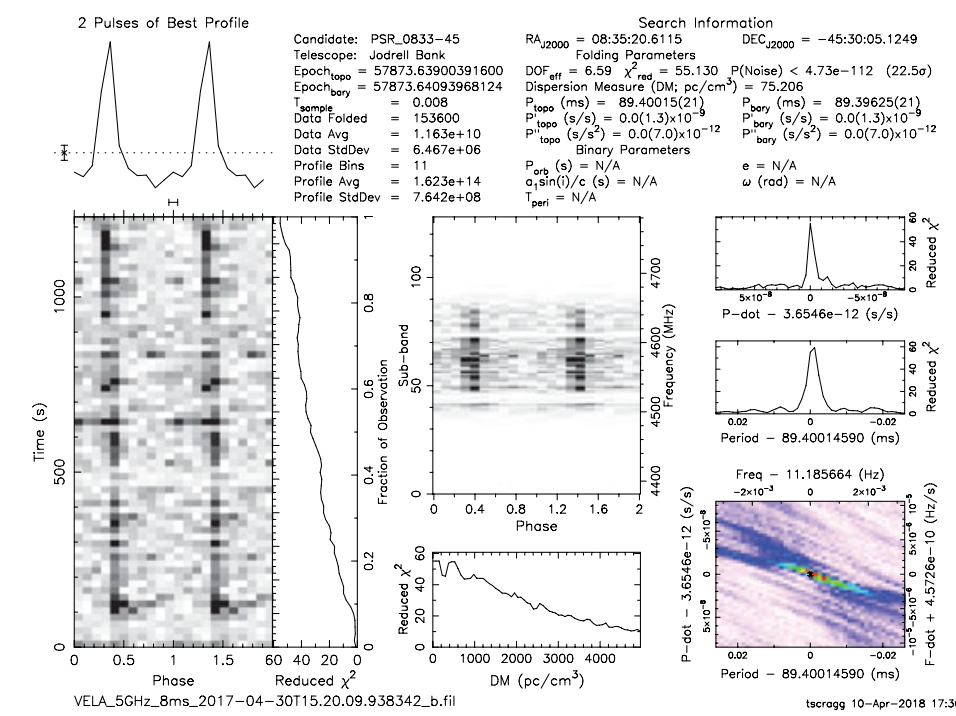

Figure 2. Detection of Vela at the GRAO, 30th April 2017.

\section{Pulsar Detection and RFI}

During a pre-commissioning visit to Ghana we observed the Vela pulsar (PSR J08354510 ) at $5 \mathrm{GHz}$, using the available spectrometer, and then processed the recorded data offline on Hebe. The integration time was short $(98 \mathrm{~s})$ and the time resolution low at $3 \mathrm{~ms}$, but the PRESTO† plot in Figure. 2 clearly shows the detection of pulses from Vela.

In our initial observations of just a few hours spread over several days, we detected the presence of some persistent RFI in both the $5 \mathrm{GHz}$ and $6.7 \mathrm{GHz}$ frequency bands. For example, when the telescope was pointing South (towards Accra), $\sim 25 \%$ of the usable bandwidth at $5 \mathrm{GHz}$ and $\sim 15 \%$ of the bandwidth at $6.7 \mathrm{GHz}$ was affected.

\section{Acknowledgement}

This publication has received funding from the European Unions Horizon 2020 research and innovation programme under grant agreement No 730562 [RadioNet].

\section{Reference}

van Straten, W. \& Bailes, M. 2011 PASA, vol 28, issue 1 pp. 1-14

$\dagger$ http://www.cv.nrao.edu/ sransom/presto/ 